\title{
A structured literature review on the interplay between emerging technologies and COVID-19 - insights and directions to operations fields
}

\author{
Maciel M. Queiroz ${ }^{1,2}$ (D) Samuel Fosso Wamba ${ }^{3}$
}

Accepted: 6 May 2021

(c) Springer Science+Business Media, LLC, part of Springer Nature 2021

\begin{abstract}
In recent years, emerging technologies have gained popularity and being implemented in different fields. Thus, critical leading-edge technologies such as artificial intelligence and other related technologies (blockchain, simulation, 3d printing, etc.) are transforming the operations and other traditional fields and proving their value in fighting against unprecedented COVID-19 pandemic outbreaks. However, due to this relation's novelty, little is known about the interplay between emerging technologies and COVID-19 and its implications to operations-related fields. In this vein, we mapped the extant literature on this integration by a structured literature review approach and found essential outcomes. In addition to the literature mapping, this paper's main contributions were identifying literature scarcity on this hot topic by operations-related fields; consequently, our paper emphasizes an urgent call to action. Also, we present a novel framework considering the primary emerging technologies and the operations processes concerning this pandemic outbreak. Also, we provided an exciting research agenda and four propositions derived from the framework, which are collated to operations processes angle. Thus, scholars and practitioners have the opportunity to adapt and advance the framework and empirically investigate and validate the propositions for this and other highly disruptive crisis.
\end{abstract}

Keywords COVID-19 $\cdot$ Emerging technologies $\cdot$ Artificial intelligence $\cdot$ Structured literature review

Maciel M. Queiroz

maciel.queiroz@docente.unip.br; macielmanoel.queiroz@mackenzie.br

Samuel Fosso Wamba

s.fosso-wamba@tbs-education.fr

1 Postgraduate Program in Business Administration, Paulista University-UNIP, Dr. Bacelar Street 1212, Sao Paulo 04026-002, Brazil

2 School of Engineering, Mackenzie Presbyterian University, Consolação Street 930, Sao Paulo 01302-000, Brazil

3 Information, Operations and Management Sciences, TBS Business School, 1 Place Alphonse Jourdain, 31068 Toulouse, France 


\section{Introduction}

The COVID-19 pandemic outbreak has caused unparallel disruptions in practically all fields and organizations' business models (Dubey, Bryde et al., 2020; Ivanov, 2020a; Pan \& Zhang, 2020; Queiroz, Ivanov et al., 2020). In consequence, traditional industries like automotive (Forbes, 2020), food supply chain (Butu et al., 2020; Singh et al., 2020), information systems and education (Dwivedi et al., 2020), medical supplies (Manero et al., 2020; Pacheco \& Laguna, 2020), hospital operations (Bauer et al., 2020; Marin-Garcia et al., 2020; Mileder et al., 2020), and the transportation sector (Baveja et al., 2020), among others, experimented exceptional disruptions and challenges (Ruel et al., 2021).

In this context, industry practitioners (Delloite, 2020; McKinsey, 2020) and scholars (Ivanov, 2020b; Remko, 2020; Sarkis et al., 2020; Sodhi et al., 2021) has agreed about the decisive role of technologies in to fight against COVID-19 and other future disruptive events and emergency situations (Amaratunga et al., 2021). More specifically, the emerging technologies (Grover et al., 2020; Queiroz, Fosso Wamba et al., 2020), also known as cutting-edge technologies, refer to a set of disruptive technologies that present fast growth, major impact, and in various cases, radical novelty, but with its potential under development (Akter et al., 2020; Rotolo et al., 2015).

In this vein, organizations and the whole society can benefit from a wide range of disruptive digital technologies (M. Gupta et al., 2021; Spanaki et al., 2021) to face an exceptional event like COVID-19. These include artificial intelligence (Belhadi et al., 2021; Fosso Wamba, Bawack, et al., 2021), blockchain (Dubey, Gunasekaran, Bryde, et al., 2020; Wamba \& Queiroz, 2020), big data analytics (Dubey, Gunasekaran, Childe, et al., 2020; Fosso Wamba, Queiroz, et al., 2020), the internet of things (A. Sinha et al., 2019), 5G (Siriwardhana et al., 2020), 3d printing (Belhouideg, 2020), virtual reality (Mao et al., 2020), augmented reality (Sahu et al., 2020), digital twin applications (Ivanov \& Dolgui, 2020), as well as the Industry 4.0 applications (Kumar \& Singh, 2021; Queiroz, Ivanov et al., 2020).

While the literature exploring COVID-19 in different operations, management, and information systems perspectives have made significant progress (Chesbrough, 2020; Dwivedi et al., 2020; Y. Li et al.,2020; Manero et al., 2020; Queiroz, Ivanov et al., 2020; Remko, 2020; Sarkis, 2020; Sodhi et al., 2021; Ivanov, 2020a, b), there is still a huge gap when we consider a systemization about the role of emerging technologies to fight against this pandemic outbreak, the lessons, insights, and directions for other future emergency situations (Choi, 2021; Ivanov, 2021).

In this line of thought, the literature dealing with the interplay between technologies approaches and COVID-19 shows a lack of a structured integration of key emerging technologies for COVID-19 control (Ivanov, 2020b; S. Kumar et al.,2021; Queiroz, Ivanov et al., 2020). For example, despite the recent, rapid increase in the number of reviews (Chowdhury et al., 2021; Ranjbari et al., 2021; Surabhi \& Anders, 2020), most of them are limited to fully explore the integration between emerging technologies and COVID-19 as a primary approach. In this regard, Surabhi and Anders (2020) presented a bibliometric analysis integrating business and management perspectives. Ranjbari et al. (2021) provided a systematic literature review with a focus on the issue of sustainability and COVID-19, and concluded by drawing an agenda considering sustainable development. In the supply chain field, Chowdhury et al. (2021) made a systematic literature review and presented interesting research opportunities on this integration.

In addition, some literature reviews have predominantly focused on only one of these elements (Choi, 2021; Queiroz, Ivanov et al., 2020). For instance, Choi (2021) presented a 
well-articulated literature analysis exploring the COVID-19 in the lens of operations management. Queiroz, Ivanov et al., (2020) explored the COVID-19 and other epidemic outbreaks using a structured literature approach and focusing on supply chain-related fields. Katsaliaki et al. (2021) provided a literature review on supply chain disruptions, including the COVID-19, but without any focus on emerging technologies.

In order to minimize this gap, our study follows a structured literature review strategy approach (Queiroz, Ivanov et al., 2020) to explore the dynamics of the relationship between emerging technologies in the COVID-19 pandemic outbreak. Thus, the following research questions (RQs) emerge:

RQ1. What is the dynamics used for publications dealing with the interplay between COVID-19 and emerging technologies?

RQ2. What technologies are being used to fight against COVID-19?

RQ3. What are the main lessons about the application of emerging technologies to emergency events such as COVID-19?

Regarding this study's main contributions, we expect to provide a well-articulated systematization about the main emerging technologies and their role in the fight against COVID19. This study intends to unlock new research streams by presenting a novel categorization and an insightful research agenda to support industry practitioners and scholars in their efforts to understand the contributions and role of key technologies applied in emergency situations.

This paper is organized as follows. Section 2 presents the methodology approach, followed by the analysis of the results in Sect. 3. In sequence, Sect. 4 points out the discussion and implications. Section 5 introduce a novel framework and research directions. Finally, Sect. 6 is dedicated to highlighting the concluding remarks and the main contributions.

\section{Methodology approach}

Following recent studies (Beydoun et al., 2019; Kapoor et al., 2018; Mishra et al., 2018), we adopted a bibliometric approach to capture the literature's dynamics regarding the interaction between emerging technologies and COVID-19. Bibliometric analysis is considered a robust approach to map a particular field by providing different metrics in order to support a more in-depth comprehension of the topic (Beydoun et al., 2019; Mishra et al., 2018; Nunes \& Pereira, 2021). Besides, we applied a structured strategy to manage a research protocol and provide new categorization to the literature (Queiroz, Ivanov et al., 2020). This mixed strategy can be considered a structured literature review by integrating these two literature review techniques (bibliometric and systematic) (Queiroz, Ivanov et al., 2020) (Table 1).

Firstly, we identified the leading and reliable, and trustworthy database to support the search. Thus, we used the Web of Science database (Clarivate Analytics, 2020) as the main source to search. Secondly, in order to organize the amount of data, we adopted two well-known software. For this reason, we employed Biblioshiny (Aria \& Cuccurullo, 2017; Queiroz, Ivanov et al., 2020) and VOSviewer (Mishra et al., 2018; Nunes \& Pereira, 2021). We selected the keywords by screening on Google Scholar different previous studies exploring COVID-19 and proposing emerging technologies to fight against this pandemic outbreak. 
Table 1 Research protocol

\begin{tabular}{ll}
\hline Dimension & Description \\
\hline Keywords & (TS = ("covid-19" OR "Covid19" OR "Covid" OR \\
& "coronavirus" OR "Sars-CoV-2" OR "SarsCoV2") \\
& AND TS = ("Internet of things" OR "IoT" OR "artificial \\
& intelligence" OR "machine learning" OR "deep learn- \\
& ing" OR 5G OR "Serverless Computing" OR block- \\
& chain OR Robotics OR Biometrics OR "3D Printing" \\
& OR "Additive Manufacturing" OR "Virtual Reality" OR \\
& "Augmented Reality" OR Drone OR "Digital twin")) \\
& 2020 \\
Timespan & SCI-EXPANDED, SSCI, A\&HCI, CPCI-S, CPCI-SSH, \\
Web of Science databases & ESCI \\
Fields & Title, Abstract, and Keywords \\
Inclusion criteria & Papers published in the WoS database \\
Complete information about the article's data & \\
Exclusion criteria & Non-English language articles \\
Incomplete information about the article & \\
Data extraction and analysis & Biblioshiny and VOSviewer \\
\hline
\end{tabular}

\section{Results analysis}

\subsection{Main information of the search}

By applying the keywords mentioned in the research protocol, we identified 1,297 papers. In sequence, we employed the inclusion and exclusion criteria, which in turn resulted in 1247 papers to analyze. Regarding some basic metrics, the average citations per paper are 3.76. A total of 3,406 keywords were used for 1,247 papers. Also, 6,473 authors appeared in these articles, and only 114 papers were single-authored papers. The average number of authors per document was 5.19. These initial numbers are highlighted in Table 2, and show an interesting interaction and contribution between the authors.

\subsection{Sources indicators}

In Table 3, we present the top 20 sources based on a number of publications (NP) and total citations (TC). We found the dominance of the journals from the computer fields, medical and medical informatics. In this vein, the first ranked was the IEEE Access $(\mathrm{NP}=57)$, a multidisciplinary and open access journal, which publishes several papers on the interplay between computer science and engineering. However, when considering the TC, the most ranked journal is the International Journal of Environmental Research and Public Health $(\mathrm{TC}=215)$, which is focused on the interactions between environmental science and medicine. While these three categories (computer fields, medical and medical informatics) dominate the most relevant sources considering the output and citations, journals from traditional areas like operations management/research, production systems, logistics, supply chain, information systems and business management do not appear in the ranking. 
Table 2 Main information

\begin{tabular}{ll}
\hline Description & Results \\
\hline Timespan & 2020 \\
Sources (Journals, Books, etc.) & 613 \\
Documents & 1247 \\
Average citations per documents & 3.763 \\
Average citations per year per doc & 2.056 \\
Document contents & \\
Author's Keywords (DE) & 3406 \\
Authors & \\
Authors & 6473 \\
Author Appearances & 7437 \\
Authors of single-authored documents & 108 \\
Authors of multi-authored documents & 6365 \\
Authors collaboration & \\
Single-authored documents & 114 \\
Documents per Author & 0.193 \\
Authors per Document & 5.190 \\
\hline
\end{tabular}

\subsection{Authors indicators}

To explore the indicators from the authors, Table 4 presents the top 20 ranked authors based on the output of papers. Also, we added the fractional authorship indicator, which computes an individual author's contributions to a group of papers published (Geunes \& $\mathrm{Su}, 2020)$. The most contributed author was Li (10), followed by Wang (8), Duong (6), and Kumar (6). It is interesting to note that four authors outperformed five papers.

\subsection{Affiliations information}

Considering the outcomes by affiliations (Table 5), the first five of the ranking were Chinese and American institutions. The most productive was Huazhong University of Science and Technology, a Chinese university, with 47 papers, followed by the Americans Icahn School of Medicine at Mount Sinai (39 papers), Harvard Medical School (32 papers), Stanford University (27 papers), and again a Chinese institution with Fudan University (26 papers). Furthermore, considering the top 20, this behavior is similar, with a few exceptions: the University of Toronto (sixth position), King Saud University (eighth), the University of Milan (eleventh), followed by the University of Oxford (twelfth), and the University of Cambridge (seventeenth).

\subsection{Information about countries production}

Regarding the countries' papers production (Table 6), the USA (1,047), China (804), India (382), Italy (360), and the United Kingdom (262) rank the top five. In this outlook, only two countries outperformed 500 papers. Moreover, the ranking comprises another North America country (Canada) that achieved great performance. While countries from Asia and Europe reached excellent participation, countries from underrepresented regions did 


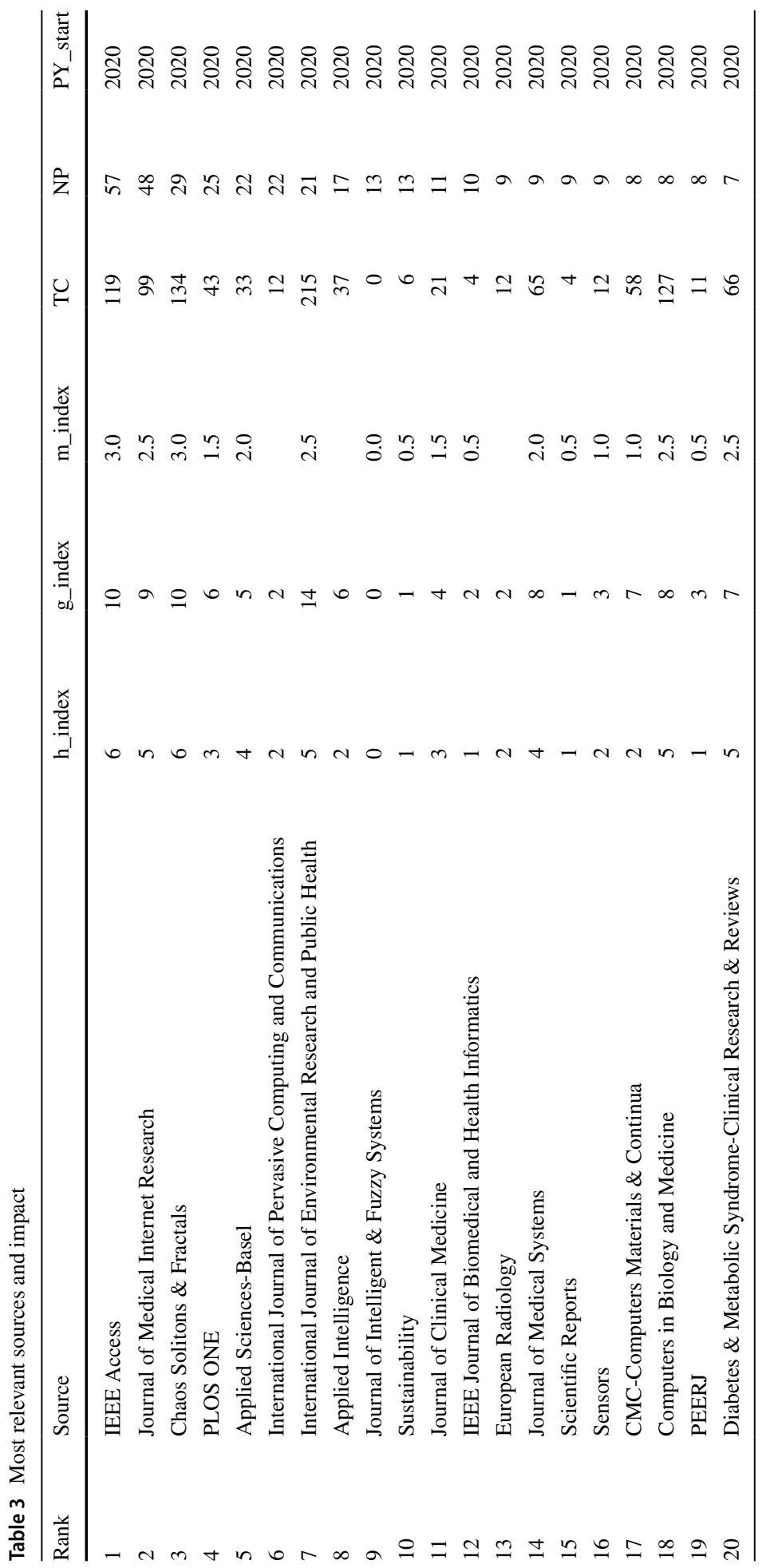


Table 4 Most relevant authors

\begin{tabular}{llll}
\hline Rank & Authors & Articles & $\begin{array}{l}\text { Articles } \\
\text { Fractional- } \\
\text { ized }\end{array}$ \\
\hline 1 & Li L & 10 & 0.74 \\
2 & Wang J & 8 & 0.91 \\
3 & Duong TQ & 6 & 0.89 \\
4 & Kumar S & 6 & 2.45 \\
5 & Das R & 5 & 0.66 \\
6 & Hossain Ms & 5 & 1.16 \\
7 & Kumar A & 5 & 1.86 \\
8 & Lee J & 5 & 0.70 \\
9 & Li Hf & 5 & 0.69 \\
10 & Liu J & 5 & 0.30 \\
11 & Peng CZ & 5 & 0.83 \\
12 & Pirouz B & 5 & 1.00 \\
13 & Sharma A & 5 & 1.39 \\
14 & Wang L & 5 & 0.37 \\
15 & Wang Yl & 5 & 1.16 \\
16 & Ye Rz & 5 & 0.83 \\
17 & Zhang Y & 5 & 0.46 \\
18 & Zhu Ts & 5 & 0.88 \\
19 & Abdulkareem KH & 4 & 0.33 \\
20 & Ali S & 4 & 0.56 \\
\hline
\end{tabular}

not appear in the top-10 list. For instance, it should be noticed the stark absence of Central Africa's countries. Surprisingly, a Latin American country, namely Brazil, achieved the eighteenth position.

\subsection{Citations per countries}

In Table 7, we consider the countries' citations. It can be seen that although China and the USA are practically tied in a number of citations, with 1,129 and 1024, respectively, the performance of the average article citations of China is better. In addition, these two countries were the only ones that outperformed 1,000 citations. The third in the rank, India, raised only 298 citations. On the one hand, China and the USA led the total citations; surprisingly, the average citations were dominated by Mauritius (40.000), Belgium (30.889), and Croatia (15.500). However, it is important to note that a small number of papers can benefit from the average article's citations.

\subsection{Top 20 papers based on the number of citations}

Table 8 shows the 20 papers most cited, their respective first authors, and the journal. The top-ranked was a systematic review and critical analysis of prediction models for COVID19. While the second paper did not focalize on any specific utilization of an AI-related 


\begin{tabular}{lll}
\hline Rank & Affiliations & Articles \\
\hline 1 & Huazhong Univ Sci and Technol & 47 \\
2 & Icahn Sch Med Mt Sinai & 39 \\
3 & Harvard Med Sch & 32 \\
4 & Stanford Univ & 27 \\
5 & Fudan Univ & 26 \\
6 & Univ Toronto & 24 \\
7 & Natl Univ Singapore & 22 \\
8 & King Saud Univ & 21 \\
9 & Zhejiang Univ & 21 \\
10 & China Med Univ & 19 \\
11 & Univ Milan & 19 \\
12 & Univ Oxford & 19 \\
13 & Shanghai Jiao Tong Univ & 18 \\
14 & Univ Hong Kong & 18 \\
15 & Johns Hopkins Univ & 16 \\
16 & Michigan State Univ & 16 \\
17 & Univ Cambridge & 16 \\
18 & Univ Penn & 16 \\
19 & Wuhan Univ & 16 \\
20 & Univ Calif Los Angeles & \\
\hline & & \\
\hline
\end{tabular}

technology, the authors highlight the role of IoT in to fight against COVID-19 and other epidemic outbreaks; in the third most ranked, the authors used machine learning integrated with different approaches to explore the mental's health of the people during COVID-19. In general, papers exploring AI techniques like machine learning, deep learning, deep neural networks, convolutional neural networks, and other data-driven mechanisms were the most popular approaches. Predicting models for diagnosis, psychological treatments, image trials, and health monitoring were the most popular issues explored. Surprisingly, we found an exception outside from the healthcare perspective. A paper exploring COVID-19 from the lens of operations and supply chains (Ivanov, 2020a) achieved the citations' seventh position. The paper focuses on the epidemic outbreaks prediction by a simulation approach, considering the supply chains. Finally, we can see that only six papers exceeded 100 citations.

\subsection{Keywords frequency-authors versus keywords plus}

In Table 9, we provide the most frequent keywords. On the left side, we have the keywords given by the authors, and on the right side, the keywords plus, which were not provided by the authors (found by an algorithm approach). Considering the authors' side, it is clear that the keywords used in the search (protocol) were the top-ranked "covid-19", "machine learning", "artificial intelligence", "deep learning", "coronavirus", etc. In addition, words that denote important operations supported by technologies like "telemedicine", "prediction", and "classification", appear in this list. Finally, other cutting-technologies like "internet of things", " $3 \mathrm{~d}$ printing", and "big data", also were protagonists. 
Table 6 Country scientific production

\begin{tabular}{llc}
\hline Rank & Country & Frequency \\
\hline 1 & USA & 1047 \\
2 & China & 804 \\
3 & India & 382 \\
4 & Italy & 360 \\
5 & United Kingdom & 262 \\
6 & Canada & 152 \\
7 & Spain & 127 \\
8 & South Korea & 126 \\
9 & Germany & 117 \\
10 & Australia & 96 \\
11 & France & 80 \\
12 & Saudi Arabia & 80 \\
13 & Singapore & 74 \\
14 & Turkey & 72 \\
15 & Iran & 71 \\
16 & Egypt & 65 \\
17 & Pakistan & 60 \\
18 & Brazil & 59 \\
19 & Netherlands & 49 \\
20 & Vietnam & 43 \\
\hline
\end{tabular}

Table 7 Most cited countries

\begin{tabular}{llrc}
\hline Rank & Country & Total Citations & $\begin{array}{c}\text { Average Arti- } \\
\text { cle Citations }\end{array}$ \\
\hline 1 & China & 1129 & 6.103 \\
2 & USA & 1024 & 4.016 \\
3 & India & 298 & 2.463 \\
4 & Belgium & 278 & 30.889 \\
5 & Canada & 277 & 6.756 \\
6 & Italy & 208 & 3.200 \\
7 & United Kingdom & 200 & 2.564 \\
8 & Germany & 181 & 7.870 \\
9 & Turkey & 159 & 6.360 \\
10 & Greece & 96 & 7.385 \\
11 & Iran & 77 & 5.133 \\
12 & Korea & 62 & 1.676 \\
13 & Netherlands & 58 & 7.250 \\
14 & Australia & 47 & 1.424 \\
15 & Brazil & 43 & 2.867 \\
16 & Mauritius & 40 & 40.000 \\
17 & Qatar & 40 & 10.000 \\
18 & Egypt & 39 & 1.696 \\
19 & Spain & 38 & 1.086 \\
20 & Croatia & 31 & 15.500 \\
\hline & & &
\end{tabular}


Regarding the keywords plus, the term "prediction" appeared in the top three, suggesting one of the main features and concerns that the emerging technologies could improve. Also, influential keywords that participated in the author's keywords, like "classification" and "artificial-intelligence", populates the top ten. Furthermore, other keywords emerged and reinforce the needs for the usage of leading-edge technologies to fight against epidemic outbreaks. The keywords were "model", "impact", "diagnosis", "risk", "management", and "design".

\subsection{Treemap dynamics}

To finalize the analysis of the keywords dynamics, in Fig. 1 we present a TreeMap taking into account the abstracts. The size rectangle denotes the frequency of the term. In this vein, "covid", "patients", "data", "pandemic", and "learning", were the most frequent topics. Regarding the emerging technologies outlook, we recognize that related topics like "data", "learning", "models", "machine", "methods", "system", "artificial”, "deep", "accuracy", "analysis", "ai", "detection", "intelligence", achieved good participation. Therefore, it bolsters the suggestion that emerging technologies play an essential role to tackle epidemic outbreaks and other emergency situations.

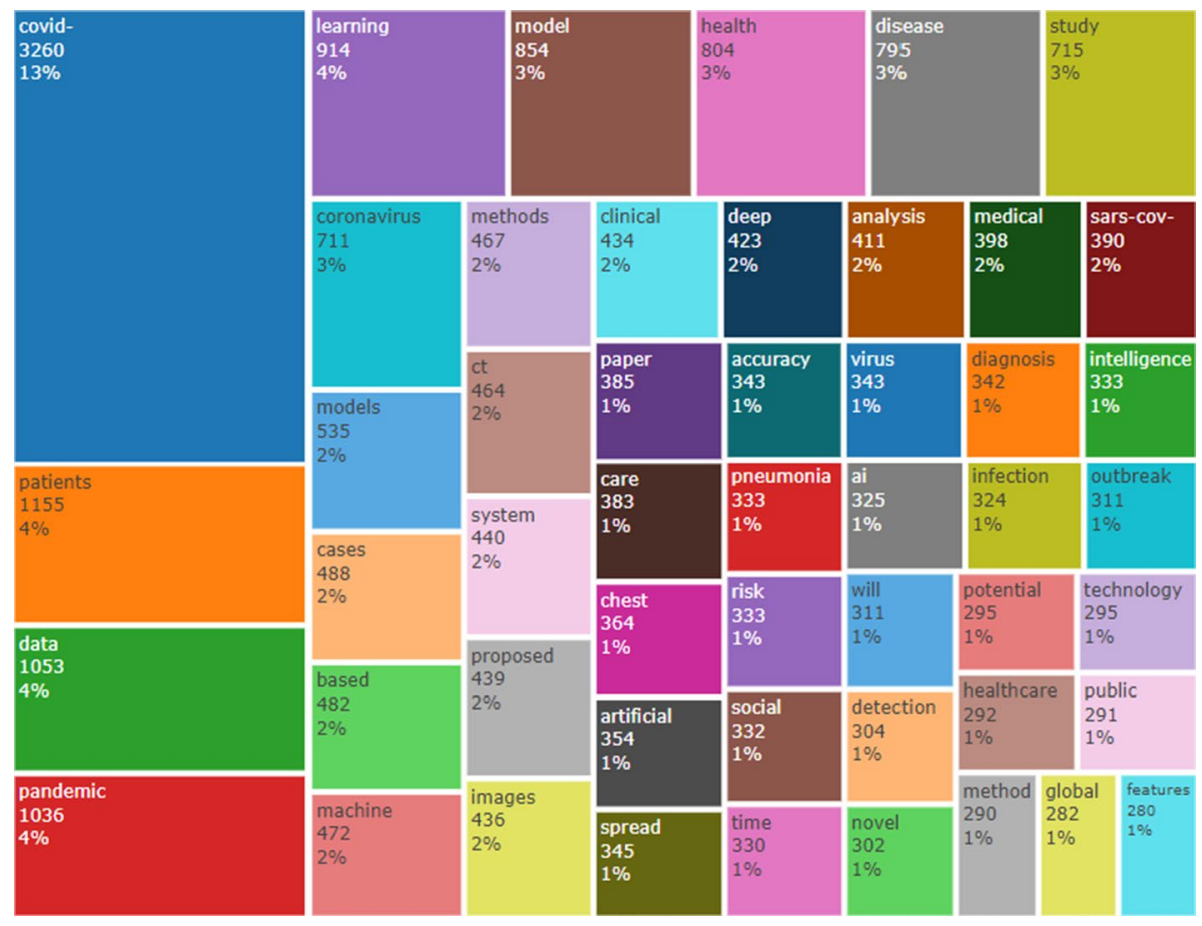

Fig. 1 TreeMap based on abstracts 


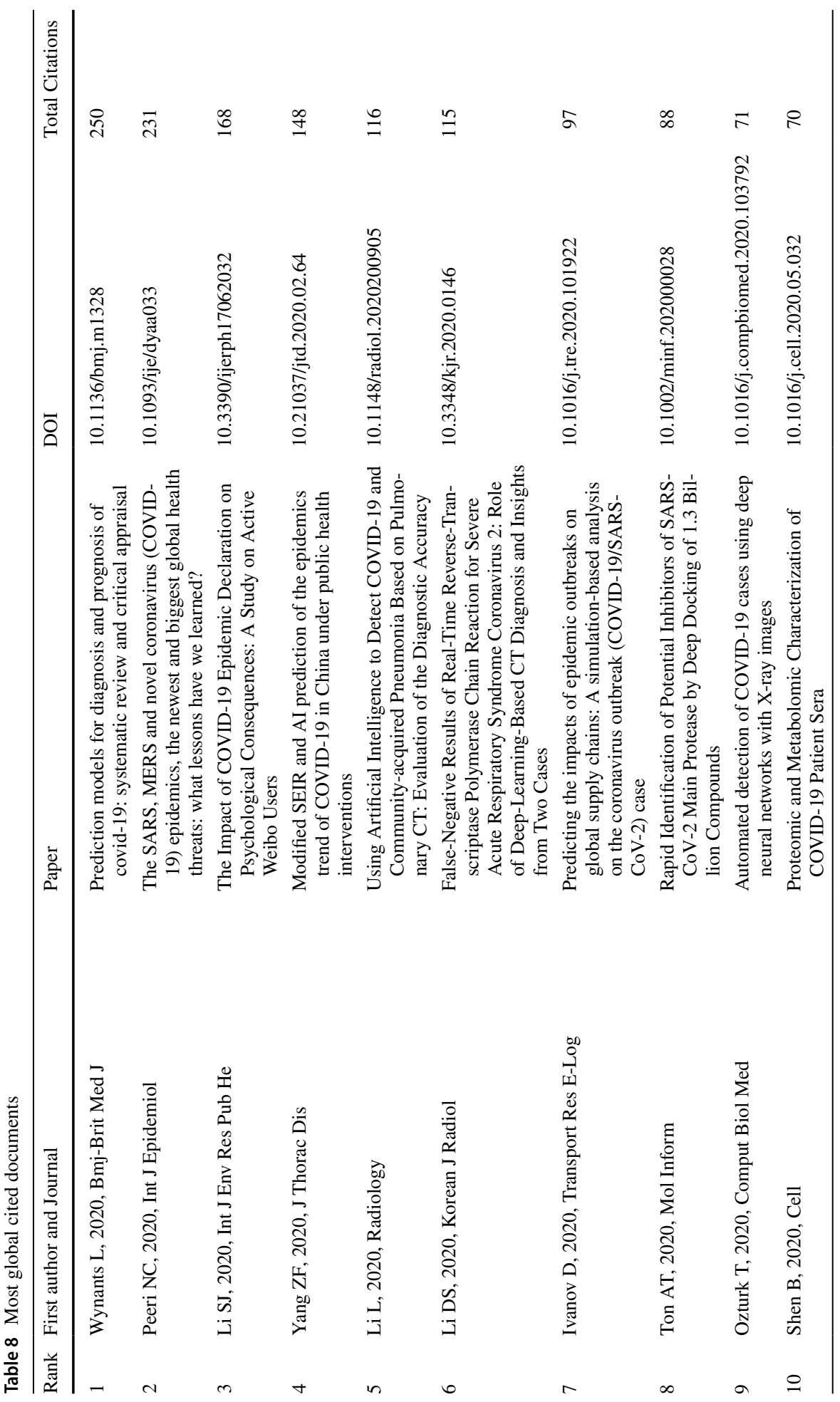




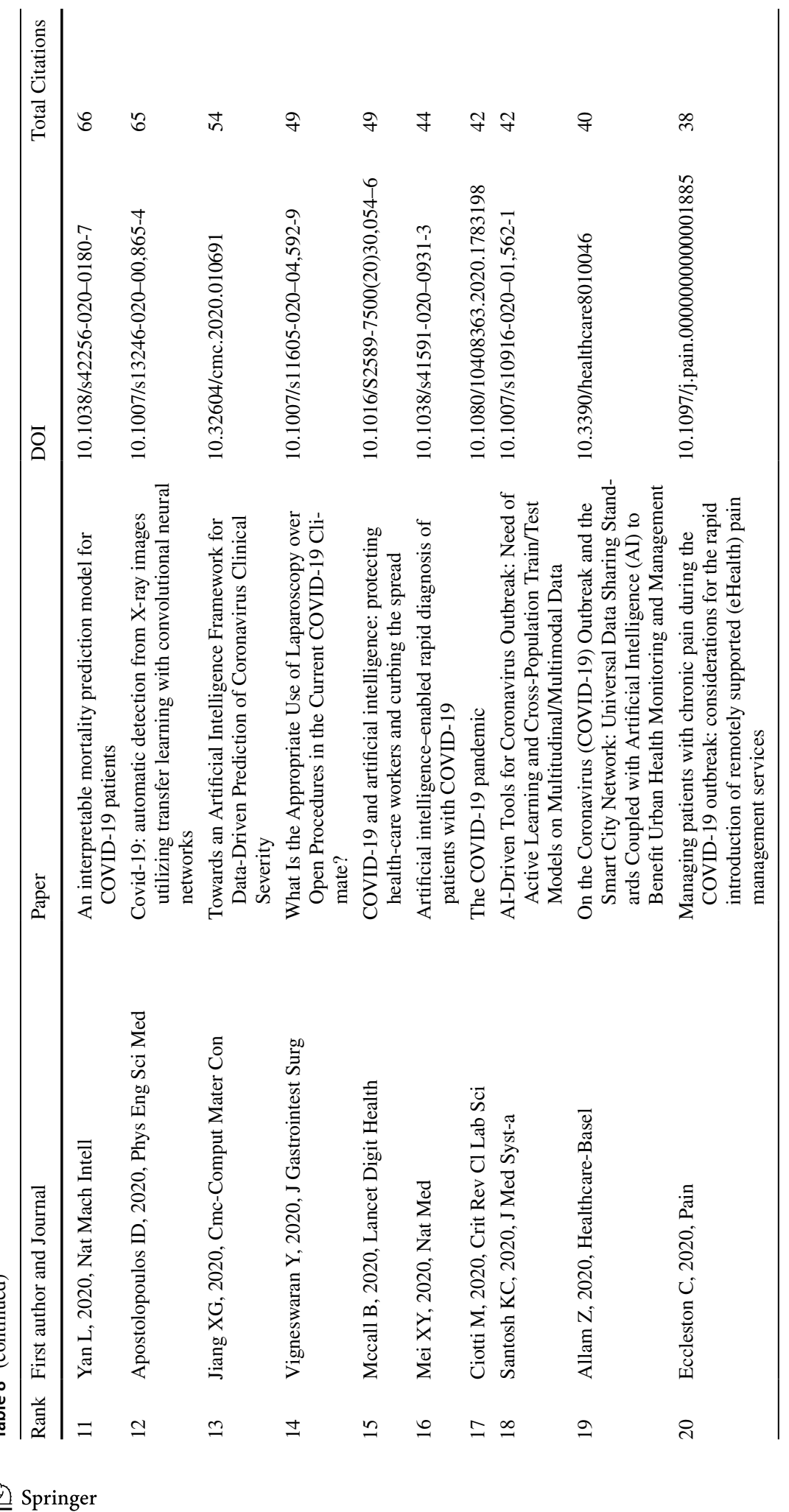


Table 9 Most frequent words

\begin{tabular}{lllll}
\hline Rank & Author's keywords & Occurrences & Keywords plus & Occurrences \\
\hline 1 & covid-19 & 677 & pneumonia & 51 \\
2 & machine learning & 181 & coronavirus & 50 \\
3 & artificial intelligence & 157 & prediction & 37 \\
4 & deep learning & 141 & china & 35 \\
5 & coronavirus & 134 & internet & 33 \\
6 & sars-cov-2 & 113 & health & 32 \\
7 & pandemic & 81 & wuhan & 32 \\
8 & computed tomography & 40 & classification & 31 \\
9 & pneumonia & 39 & artificial-intelligence & 30 \\
10 & learning & 38 & system & 30 \\
11 & internet of things & 37 & disease & 27 \\
12 & telemedicine & 36 & model & 27 \\
13 & lung & 31 & impact & 25 \\
14 & 3d printing & 30 & outbreak & 25 \\
15 & big data & 30 & covid-19 & 24 \\
16 & prediction & 26 & diagnosis & 24 \\
17 & classification & 24 & risk & 23 \\
18 & diseases & 24 & management & 22 \\
19 & intelligence & 24 & sars & 22 \\
20 & artificial & 22 & design & 21 \\
\hline
\end{tabular}

\subsection{Cluster analysis}

We performed a cluster analysis to explore the topic's similarities characteristics (Hosseini \& Ivanov, 2020; Kafeza et al., 2020). In order to ensure reliability and replicability, Table 10 highlights the protocol adopted. Thus, we found six interesting clusters (Fig. 2).

In this respect, cluster one (red) is a mixed cluster, with emerging technologies (AI, augmented reality, big data, blockchain, IoT, robotics, simulation, virtual reality, etc.), operations medical approaches (contact tracing, digital health, telehealth), and business management (framework, innovation, management). Cluster 2 (green), is governed by deep learning and other AI approaches (convolutional neural network, neural network) applied mainly in the diagnosis process, diseases, image segmentation, recognition, segmentation,

Table 10 Cluster protocol

\begin{tabular}{ll}
\hline Type of analysis & Co-occurrence \\
\hline Unit of analysis & All keywords \\
Counting type & Full counting \\
Minimum number of occurrences of a keyword & 9 \\
Meet the threshold & 123 \\
\hline
\end{tabular}




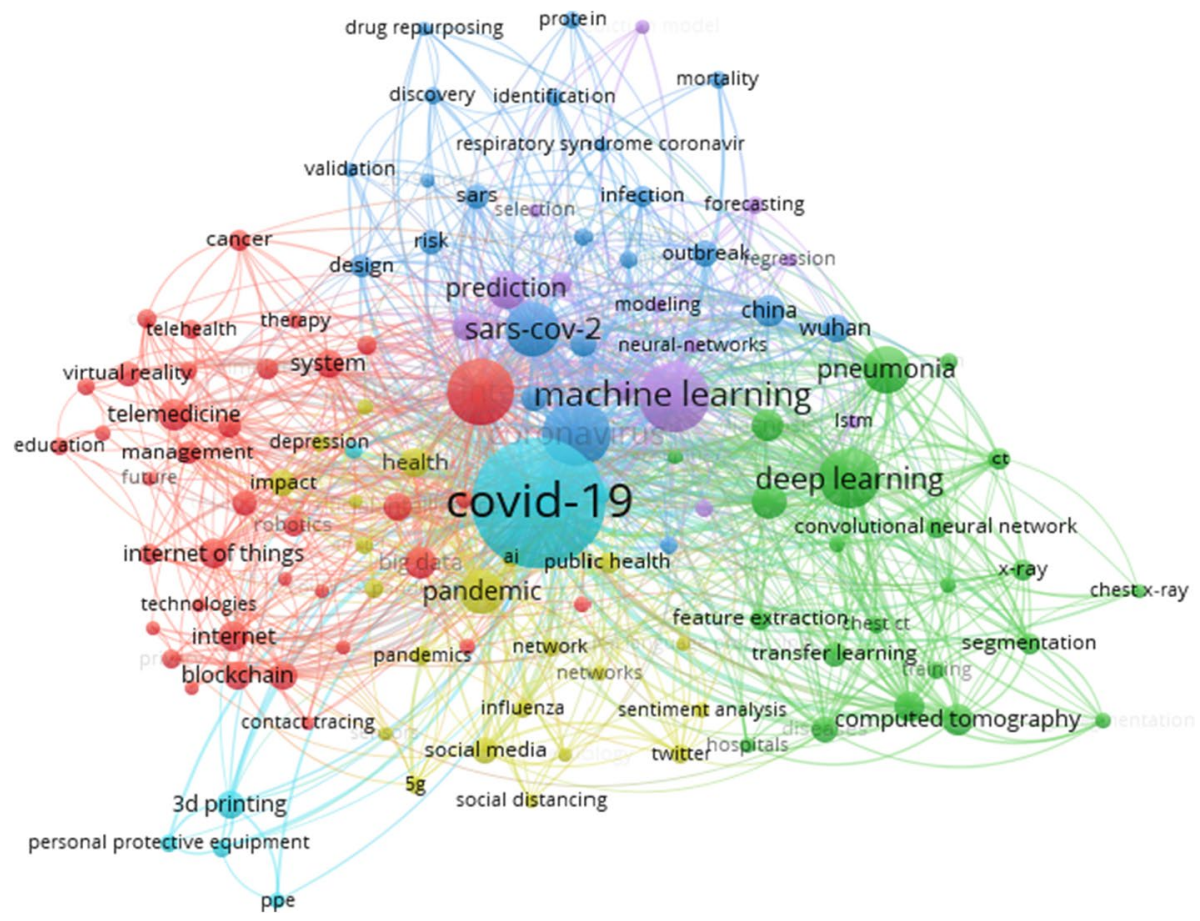

Fig. 2 Cluster analysis

$\mathrm{x}$-ray, etc. Cluster 3 (dark blue) is dedicated to sars-cov-2 dynamics (identification, infection, mortality, outbreak, etc.). In sequence, cluster 4 (yellow) concentrates on social network approaches (networks, sentiment analysis, social media, Twitter, etc.) and other technology approaches (AI, natural language processing, 5G) to support the evolution of the pandemic outbreak. Next, cluster 5 (purple) is concentrated on technical aspects of the technologies (algorithm, forecasting, machine learning, model, neural networks, prediction, long short-term memory (LSTM), etc.). Lastly, cluster 6 (light blue) is focused on equipment supply (3d printing/additive manufacturing, personal protective equipment, etc.).

\section{Discussion and implications}

Following the main aim of this study, regarding the investigation of the relationship between emerging technologies and COVID-19 by the lens of operations-related fields, our structured literature review revealed interesting behavior. Firstly, considering one year of time horizon, we found 1,247 papers, and 6,473 authors; this is an impressive output. The papers resulting from a collaboration between several authors suggest an interesting effort between authors and countries to provide insights against this pandemic. Thus, resulting in a good average citation per article (3.763).

Given the journals' performance, while journals from the computer, medical, and medical informatics fields dominate the top 20 journals from production and operations management fields do not appear in the ranking. Thus, it can be seen as an alert for these fields 
to engage the community on this topic. From the author's productivity side, four authors exceeded five papers - Li (10), Wang (8), Duong (6), and Kumar (6). Regarding the most productive institutions, China and the USA dominate the list. The same behavior was found in most cited countries' analysis. However, taking into account the average article citations, other countries appear more ranked (Mauritius, Belgium, and Croatia). It could be seen that due to a large number of papers from the USA and China, the average citation is minimized.

Regarding the performance of the top-cited papers, we found that machine learning, deep learning, deep neural networks, convolutional neural networks, and data-driven were the most popular techniques (Apostolopoulos \& Mpesiana, 2020; Jiang et al., 2020; D. Li et al., 2020; Wynants et al., 2020; Yan et al., 2020). Although these techniques were predominantly applied in healthcare-related themes, we found an outlier representing the production and operations management field. A paper investigating the dynamics of the epidemic outbreaks on supply chains (Ivanov, 2020a), supported by the simulation approach, ranked in the seventh position.

The keywords analysis revealed interesting behavior in the literature. By comparing the authors versus keywords plus, we found similarities and some different words. From the author's lens, besides the popularity of the words used in our search (machine learning, artificial intelligence, deep learning, etc.), no keywords concerning a direct connection to operations and production management fields were found. Considering the keywords plus, the ranking was dominated by related-pandemic words. Furthermore, "prediction" appeared in both rankings, showing the need to use some emerging technologies to improve the response and the operations as a whole.

In order to explore other perspectives from the dynamics of the word, we performed a TreeMap analysis based on the abstracts. While we found that AI-related technologies reached good participation in the frequency, words that evidence a direct application to the production and operations management were scarce. However, it is important to note that there was an indirect connection of the keywords when it considered the angle from healthcare operations. For instance, "data", "learning", "models", "methods", "system", "artificial", "accuracy", "analysis", "detection", etc.

In relation to the cluster analysis, we found six clusters. The first, although being miscellaneous, it was dominated by emerging technologies that are related to AI (virtual reality, augmented reality, blockchain, big data, IoT, robotics, simulation, etc.), that are used to support operations medical approaches (contact tracing, digital health, telehealth). We found that cluster 2 was focused on deep learning and other AI approaches (convolutional neural network, neural network) to operations related to diagnosis, recognition diseases, etc. While cluster 3 did not present any production and operations perspective, cluster 4 emphasized social networks and other technologies like $5 \mathrm{G}$ to follow the evolution of the pandemic. Finally, cluster 5 emphasized the technical approaches (i.e., algorithms) of the emerging technologies, and cluster 6 concentrated on medical supply using technologies (i.e., $3 \mathrm{~d}$ printing).

\subsection{Theoretical implications}

Our results unveiled intriguing behavior regarding the dynamics of the interplay between emerging technologies and COVID-19. Firstly, while journals from the computer, medical and medical informatics literature were protagonists in exploring emerging technologies 
(Hao-Chih et al., 2020; Jiang et al., 2020; McCall, 2020; Ozturk et al., 2020), there is a huge gap concerning journals from production and operations-related fields, regarding the output's perspective. In this vein, an exception was Ivanov (2020a), with his paper "Predicting the impacts of epidemic outbreaks on global supply chains: A simulation-based analysis on the coronavirus outbreak (COVID-19/SARS-CoV-2) case", published on Transportation Research Part E. Thus, it suggests a more engagement by the scholars and journals of these fields to advance on this hot topic, increasing the output and the influence of the papers published.

From the emerging technologies angle, AI technologies like machine learning and deep learning were the most common approaches (D. Li et al., 2020; S. Li et al., 2020) but mainly applied to diagnosis, telemedicine, and prediction on hospital's operations. Other disruptive technologies like blockchain, virtual reality, 5G, 3d printing, big data, simulation, and IoT were found less frequent that the aforementioned AI technologies, but all can be integrated with AI technologies in order to leverage the power of the solutions to fight against epidemic outbreaks. Surprisingly, words related to "serverless computing", "biometrics", "drone", and "digital twin" did not appear in the keywords or cluster analysis. Thus, it suggests that these topics are embroidery in this perspective (AI and COVID19), mainly regarding the production and operations fields.

Furthermore, in Sect. 5, we provide valuable research directions considering different and the most adherent operations approaches and organizational theories that can be used to explore the interplay between emerging technologies, COVID-19 and other emergency situations.

\subsection{Practical implications}

Regarding the practical and managerial implications, our study highlights that managers and practitioners should consider the adoption of AI-related technologies to improve their operations, independently of the segment of the organization (Dwivedi et al., 2019). For instance, machine learning, deep learning, and data-driven are powerful approaches to improve the prediction's capabilities of the organizations and, consequently, develop strategies to anticipate, respond, and improve their operations during and after complex crisis such as COVID-19. Moreover, other leading-edge technologies like blockchain, 5G, $3 \mathrm{~d}$ printing and IoT, represent opportunities to managers integrate into their operations in order to improve the traceability of the goods, as well as replace some of the essential equipment by employing $3 \mathrm{~d}$ printing.

Besides, as we consider that it is not trivial to integrate various emerging technologies under a holistic framework as an attempt to better inform practitioners, the next section proposes a framework that highlights key emerging technologies and their usage level, as well as some activities that could be supported by them in relation to emergency situations. Furtermore, the digital twin approach (Ivanov \& Dolgui, 2020) appears as another valuable approach to combining different technologies in the same framework for the same purpose. 


\section{Framework and research directions}

Based on the findings and the lack of literature, in this section, we provide a novel framework (Fig. 3) considering the interplay between AI, complex emergency situations like COVID-19, and the integration with production and operations-related fields. The framework has four AI perspectives.

First, the "Emerging technologies relative mature level", which is related to technologies that are already being used in different COVID-19 operations through the organization's network. In this category, machine learning, deep learning and big data analytics have been used to improve the predictions/forecasting regarding diagnosis, thus supporting the optimization of the resources planning (Allam \& Jones, 2020; Jiang et al., 2020; D. Li et al., 2020; S. Li et al.,2020).

The second category is the "Emerging technologies early use level", which is about the technologies required at the first stage of operations focused on COVID-19. For example, of the use of simulation techniques (Ivanov, 2020a) contributes to improving on supply chain, thereby enhancing the effectiveness of the achieved responses. This is partcularly true with $3 \mathrm{~d}$ printing, the use of which enables a quick replacement of critical medical supplies (Manero et al., 2020).

The third category is compounded by "Emerging technologies awareness level", that is, technologies with a high potential to support operations in pandemic and other types of crisis, but managers and practitioners are still gaining knowledge about its applications in operations. This category has emerging technologies like digital twin (Ivanov, 2020a), 5G (Allam \& Jones, 2020), among others.

The "Emerging technologies operations processes view" forms the last category. It encompasses the main operations that are enabled by the AI (emerging)-related technologies. Hence, we point out that with the use of the technologies from the aforementioned categories, processes related to contact tracing, telemedicine/telehealth, diagnosis, recognition, drug repurposing, forecasting, and production of critical supplies, can be significantly improved.

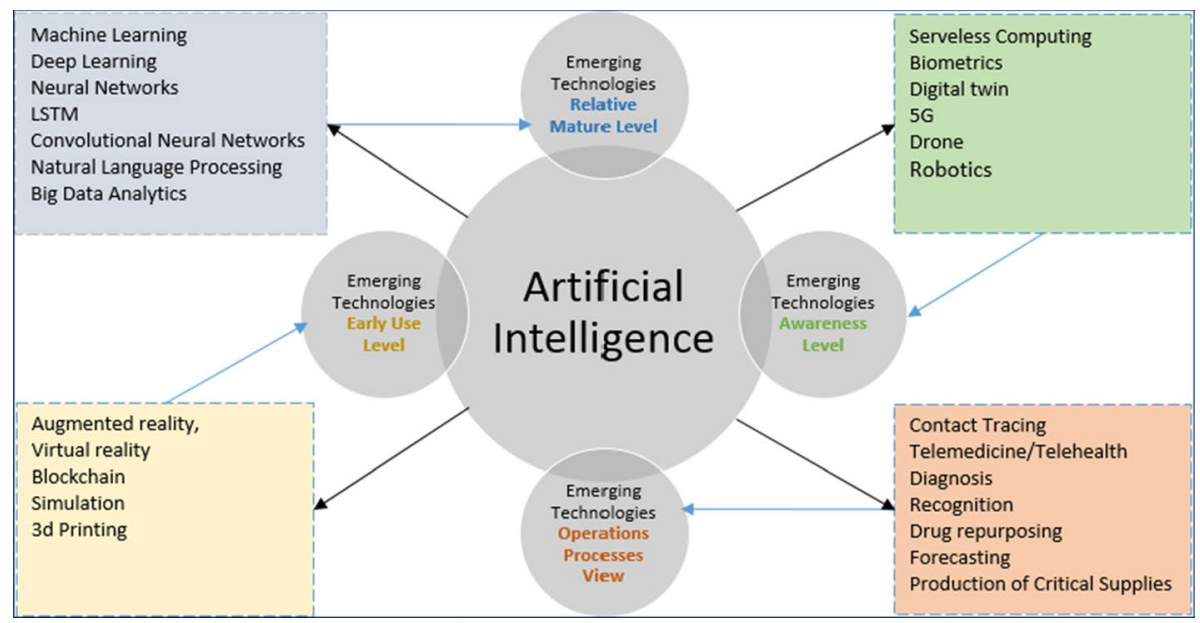

Fig. 3 Emerging technologies framework 


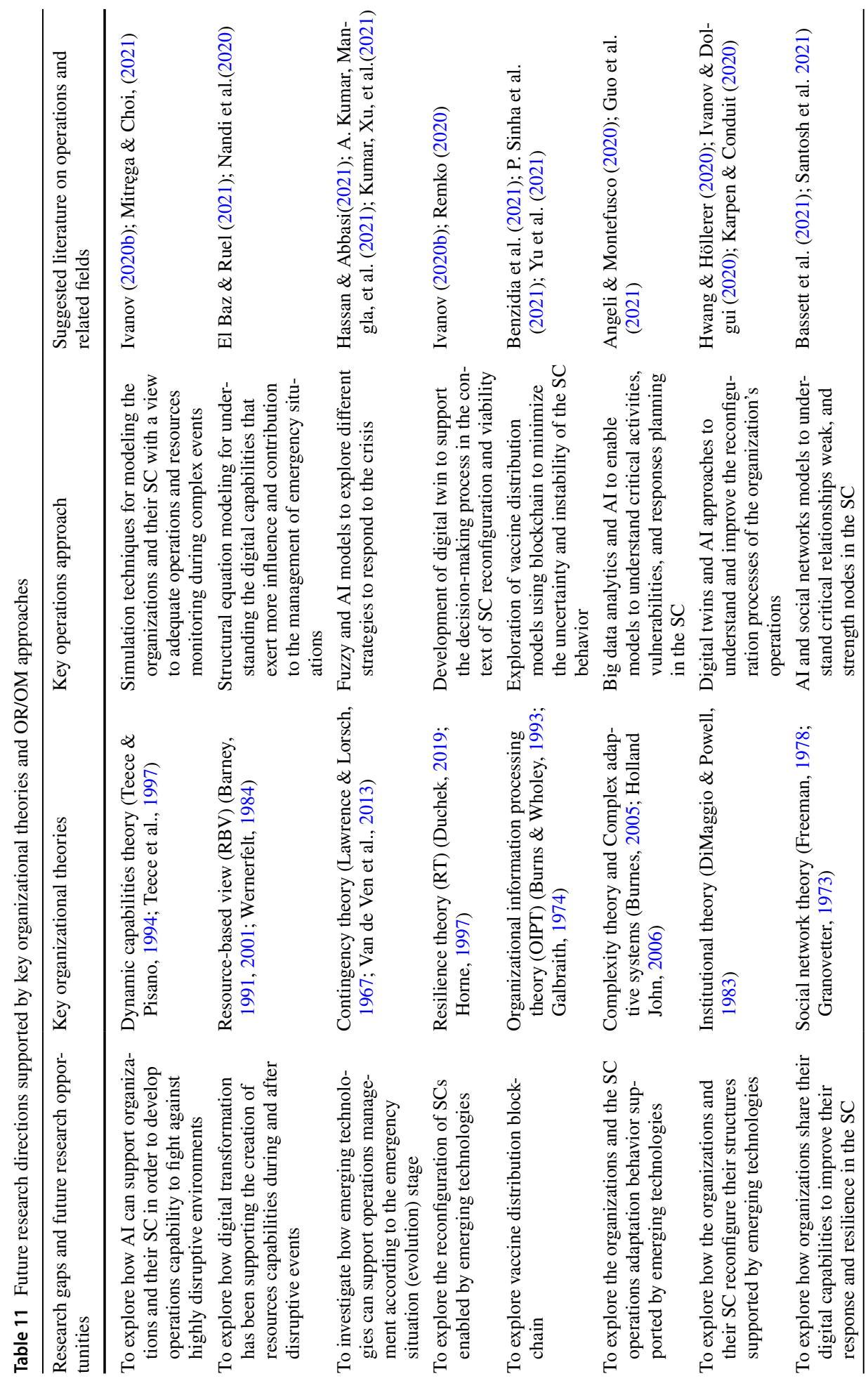




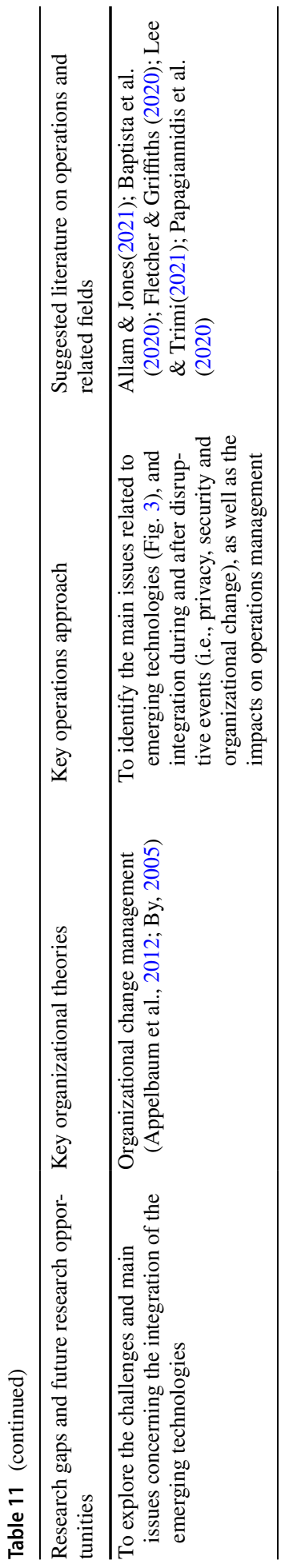


In order to better scrutinize this framework and provide valuable research directions, we derived four intriguing propositions that could be empirically investigated.

\section{Proposition 1 'Emerging technologies relative mature level' is positively associated with the operations' agility during and after a prolonged emergency situation. \\ Proposition 2 'Emerging technologies early use level' is positively associated with the operations adaptability during and after a prolonged emergency situation. \\ Proposition 3 'Emerging technologies awareness level' can positively or negatively affect the operations performance during and after a prolonged emergency situation. \\ Proposition 4 'Emerging technologies operations processes view' is positively associated with the organization's capabilities in using different technologies to sup- port their operations during and after a prolonged emergency situation.}

\subsection{Research agenda for COVID-19 and emerging technologies}

Based on S. Gupta et al. (2019) and Queiroz, Ivanov et al., (2020a), this section shows the emergence of integrative research directions in relation to emerging technologies, COVID19 and key theories. Besides, it examines the integration challenges, privacy, security and organizational change management issues with regard to the application of these emerging technologies (Hensmans, 2021; Wilson, 2020). In this vein, in Table 11 we report important research gaps concerning emerging technologies and digital transformation in the COVID-19 outlook. Moreover, in order to support scholars and practitioners, we suggest classic organizational theories, key operations approaches, and some recent literature on operations and related fields.

\subsection{Limitations}

This study harbors a number of limitations. The first is related to the unprecedented characteristics of the COVID-19 pandemic outbreak, which leads to some natural delays in some fields, with possible impacts on the journals' output concerning this topic. As a result, the search on the databases can also be affected. This is why we could not be able to obtain papers from important journals in due time. This negative impact can be upset if scholars and practitioners further use $\mathrm{AI}$ and big data techniques to monitor the literature on the interplay between emerging technologies and COVID-19. The second limitation derives from the fact that the keywords used for the search may not have considered some potential papers (Mishra et al., 2018), for one reason or the other. We suggest future literature reviews on this topic to expand the keywords to use in the databases. The third limitation comes from our using only the Web of Science database to perform the search, as multiple sources of data would have inevitably led to more convincing results. By combining the Web of Science database with other databases including Scopus, future research will definitely fill such a gap. The various research avenues outlined from these limitations, coupled with the proposed framework and its propositions, represent a significant opportunity to advance the literature, especially as regards the production and operations-related fields. Furthermore, managers and practitioners are henceforth offered the opportunity to explore our framework in order to better grasp how emerging technologies get integrated with their firms' operations. 


\section{Concluding remarks and contributions}

In this study, we explored the interplay between emerging technologies and COVID-19 by means of a structured literature review, taking into account the operations-related fields. Thus, our findings showed that cutting-edge AI technologies like machine learning and deep learning are the most popular approaches to support operations in this pandemic outbreak, but that other related technologies-like big data analytics, blockchain, simulation, AR/VR, 3d printing, etc.- - are also increasingly since the outbreak of COVID-19. Findings also indicate that a group of leading-edge technologies (i.e., digital twin, 5G, drones, etc.) remain at their infancy stage when it comes to interventions or applications in emergency situations. Similarly, it appears that all these technologies, together with more others, are mainly used in operations related to contact tracing, telemedicine, diagnosis, drug repurposing, forecasting, etc.

In terms of contributions, this study appears as one of the first papers that have mapped the literature exploring key emerging technologies in the COVID-19 outlook. Secondly, we found that traditional journals featured very few papers dealing with the production and operations fields. This therefore reinforces the need for scholars and practitioners to call for action so that this gap be filled. Thirdly, we provided a novel framework that synthesizes the main emerging technologies that can be used in the context of a huge epidemic outbreak. Fourthly, the four propositions derived from the framework can be a rich avenue for scholars and practitioners to investigate and empirically validate the role of emerging technologies in emergency situations. Lastly, we suggest other exciting future research directions supported by key organizational theories and OR/OM approaches.

\section{References}

Akter, S., Michael, K., Rajib, M., Mccarthy, G., \& Rahman, M. (2020). Transforming business using digital innovations: The application of AI, blockchain, cloud and data analytics. Annals of Operations Research. https://doi.org/10.1007/s10479-020-03620-w

Allam, Z., \& Jones, D. S. (2020). On the Coronavirus (COVID-19) outbreak and the smart city network: universal data sharing standards coupled with Artificial Intelligence (AI) to benefit urban health monitoring and management. Healthcare, 8(1), 46. https://doi.org/10.3390/healthcare8010046

Allam, Z., \& Jones, D. S. (2021). Future (post-COVID) digital, smart and sustainable cities in the wake of 6G: Digital twins, immersive realities and new urban economies. Land Use Policy. https://doi. org/10.1016/j.landusepol.2020.105201

Amaratunga, D., Cabrera, J., Ghosh, D., Katehakis, M. N., Wang, J., \& Wang, W. (2021). Socio-economic impact on COVID-19 cases and deaths and its evolution in New Jersey. Annals of Operations Research. https://doi.org/10.1007/s10479-021-03941-4

Angeli, F., \& Montefusco, A. (2020). Sensemaking and learning during the Covid-19 pandemic: A complex adaptive systems perspective on policy decision-making. World Development. https://doi.org/ 10.1016/j.worlddev.2020.105106

Apostolopoulos, I. D., \& Mpesiana, T. A. (2020). Covid-19: Automatic detection from X-ray images utilizing transfer learning with convolutional neural networks. Physical and Engineering Sciences in Medicine, 43(2), 635-640. https://doi.org/10.1007/s13246-020-00865-4

Appelbaum, S. H., Habashy, S., Malo, J., \& Shafiq, H. (2012). Back to the future: Revisiting Kotter's 1996 change model. Journal of Management Development, 31(8), 764-782. https://doi.org/10. $1108 / 02621711211253231$

Aria, M., \& Cuccurullo, C. (2017). Bibliometrix : An R-tool for comprehensive science mapping analysis. Journal of Informetrics, 11(4), 959-975. https://doi.org/10.1016/j.joi.2017.08.007 
Baptista, J., Stein, M. K., Klein, S., Watson-Manheim, M. B., \& Lee, J. (2020). Digital work and organisational transformation: Emergent Digital/Human work configurations in modern organisations. Journal of Strategic Information Systems. https://doi.org/10.1016/j.jsis.2020.101618

Barney, J. (1991). Firm resources and sustained competitive advantage. Journal of Management, 17(1), 99-120. https://doi.org/10.1177/014920639101700108

Barney, J. B. (2001). Resource-based theories of competitive advantage: A ten-year retrospective on the resource-based view. Journal of Management, 27(6), 643-650. https://doi.org/10.1016/S01492063(01)00115-5

Bassett, H. R., Lau, J., Giordano, C., Suri, S. K., Advani, S., \& Sharan, S. (2021). Preliminary lessons from COVID-19 disruptions of small-scale fishery supply chains. World Development. https://doi.org/10. 1016/j.worlddev.2021.105473

Bauer, J., Brüggmann, D., Klingelhöfer, D., Maier, W., Schwettmann, L., Weiss, D. J., \& Groneberg, D. A. (2020). Access to intensive care in 14 European countries: A spatial analysis of intensive care need and capacity in the light of COVID-19. Intensive Care Medicine. https://doi.org/10.1007/ s00134-020-06229-6

Baveja, A., Kapoor, A., \& Melamed, B. (2020). Stopping Covid-19: A pandemic-management service value chain approach. Annals of Operations Research, 289(2), 173-184. https://doi.org/10.1007/ s10479-020-03635-3

Belhadi, A., Mani, V., Kamble, S. S., Khan, S. A. R., \& Verma, S. (2021). Artificial intelligence-driven innovation for enhancing supply chain resilience and performance under the effect of supply chain dynamism: An empirical investigation. Annals of Operations Research. https://doi.org/10.1007/ s10479-021-03956-X

Belhouideg, S. (2020). Impact of 3D printed medical equipment on the management of the Covid19 pandemic. International Journal of Health Planning and Management, 35(5), 1014-1022. https://doi.org/ 10.1002/hpm.3009

Benzidia, S., Makaoui, N., \& Bentahar, O. (2021). The impact of big data analytics and artificial intelligence on green supply chain process integration and hospital environmental performance. Technological Forecasting and Social Change. https://doi.org/10.1016/j.techfore.2020.120557

Beydoun, G., Abedin, B., Merigó, J. M., \& Vera, M. (2019). Twenty years of information systems frontiers. Information Systems Frontiers, 21(2), 485-494. https://doi.org/10.1007/s10796-019-09925-X

Burnes, B. (2005). Complexity theories and organizational change. International Journal of Management Reviews, 7(2), 73-90. https://doi.org/10.1111/j.1468-2370.2005.00107.x

Burns, L. R., \& Wholey, D. R. (1993). Adoption and abandonment of matrix management programs: Effects of organizational characteristics and interorganizational networks. Academy of Management Journal Academy of Management., 36(1), 106-138. https://doi.org/10.2307/256514

Butu, A., Brumă, I. S., Tanasă, L., Rodino, S., Vasiliu, C. D., Doboș, S., \& Butu, M. (2020). The impact of COVID-19 crisis upon the consumer buying behavior of fresh vegetables directly from local producers. Case study: The quarantined area of Suceava County, Romania. International Journal of Environmental Research and Public Health, 17(15), 1-25. https://doi.org/10.3390/ijerph17155485

By, R. T. (2005). Organisational change management: A critical review. Journal of Change Management, 5(4), 369-380. https://doi.org/10.1080/14697010500359250

Chesbrough, H. (2020). To recover faster from Covid-19, open up: Managerial implications from an open innovation perspective. Industrial Marketing Management, 88, 410-413. https://doi.org/10.1016/j. indmarman.2020.04.010

Choi, T. M. (2021). Fighting against COVID-19: What operations research can help and the sense-andrespond framework. Annals of Operations Research. https://doi.org/10.1007/s10479-021-03973-w

Chowdhury, P., Paul, S. K., Kaisar, S., \& Moktadir, M. A. (2021). COVID-19 pandemic related supply chain studies: A systematic review. Transportation Research Part E: Logistics and Transportation Review. https://doi.org/10.1016/j.tre.2021.102271

Clarivate Analytics. (2020). Web of Science. https://clarivate.com/webofsciencegroup/solutions/web-ofscience/

Delloite. (2020). COVID-19: Shaping the future through digital business - Leveraging technology to support the recovery and produce lasting change. https://www2.deloitte.com/global/en/pages/about-deloitte/ articles/covid-19/shaping-the-future-through-digital-business.html

DiMaggio, P. J., \& Powell, W. W. (1983). The Iron cage revisited: Institutional isomorphism and collective rationality in organizational fields. American Sociological Review, 48(2), 147. https://doi.org/10. $2307 / 2095101$

Dubey, R., Bryde, D. J., Foropon, C., Graham, G., Giannakis, M., \& Mishra, D. B. (2020). Agility in humanitarian supply chain: An organizational information processing perspective and relational view. Annals of Operations Research. https://doi.org/10.1007/s10479-020-03824-0 
Dubey, R., Gunasekaran, A., Bryde, D. J., Dwivedi, Y. K., \& Papadopoulos, T. (2020b). Blockchain technology for enhancing swift-trust, collaboration and resilience within a humanitarian supply chain setting. International Journal of Production Research. https://doi.org/10.1080/00207543.2020.1722860

Dubey, R., Gunasekaran, A., Childe, S. J., Bryde, D. J., Giannakis, M., Foropon, C., Roubaud, D., \& Hazen, B. T. (2020c). Big data analytics and artificial intelligence pathway to operational performance under the effects of entrepreneurial orientation and environmental dynamism: A study of manufacturing organisations. International Journal of Production Economics., 226(October 2019), 107599. https:// doi.org/10.1016/j.ijpe.2019.107599

Duchek, S. (2019). Organizational resilience: A capability-based conceptualization. Business Research, 13(1), 215-246. https://doi.org/10.1007/s40685-019-0085-7

Dwivedi, Y. K., Hughes, D. L., Coombs, C., Constantiou, I., Duan, Y., Edwards, J. S., Gupta, B., Lal, B., Misra, S., Prashant, P., Raman, R., Rana, N. P., Sharma, S. K., \& Upadhyay, N. (2020). Impact of COVID-19 pandemic on information management research and practice: Transforming education, work and life. International Journal of Information Management. https://doi.org/10.1016/J.IJINF OMGT.2020.102211

Dwivedi, Y. K., Hughes, L., Ismagilova, E., Aarts, G., Coombs, C., Crick, T., Duan, Y., Dwivedi, R., Edwards, J., Eirug, A., Galanos, V., Ilavarasan, P. V., Janssen, M., Jones, P., Kar, A. K., Kizgin, H., Kronemann, B., Lal, B., Lucini, B., \& Williams, M. D. (2019). Artificial Intelligence (AI): Multidisciplinary perspectives on emerging challenges, opportunities, and agenda for research, practice and policy. International Journal of Information Management. https://doi.org/10.1016/j.ijinfomgt.2019. 08.002

El Baz, J., \& Ruel, S. (2021). Can supply chain risk management practices mitigate the disruption impacts on supply chains' resilience and robustness? Evidence from an empirical survey in a COVID-19 outbreak era. International Journal of Production Economics. https://doi.org/10.1016/j.ijpe.2020.107972

Fletcher, G., \& Griffiths, M. (2020). Digital transformation during a lockdown. International Journal of Information Management. https://doi.org/10.1016/j.ijinfomgt.2020.102185

Forbes. (2020). The Coronavirus' Impact On The Global Automotive Supply Chain. https://www.forbes. com/sites/laurieharbour1/2020/03/13/the-coronavirus-impact-on-the-global-automotive-supplychain/?sh=1317691444ec. Accessed 10 Dec 2020

Fosso Wamba, S., Bawack, R. E., Guthrie, C., Queiroz, M. M., \& Carillo, K. D. A. (2021). Are we preparing for a good AI society? A bibliometric review and research agenda. Technological Forecasting and Social Change, 164, 1-27. https://doi.org/10.1016/j.techfore.2020.120482

Fosso Wamba, S., Queiroz, M. M., Wu, L., \& Sivarajah, U. (2020). Big data analytics-enabled sensing capability and organizational outcomes: Assessing the mediating effects of business analytics culture. Annals of Operations Research. https://doi.org/10.1007/s10479-020-03812-4

Freeman, L. C. (1978). Centrality in social networks conceptual clarification. Social Networks, 1(3), 215239. https://doi.org/10.1016/0378-8733(78)90021-7

Galbraith, J. R. (1974). Organization design: An information processing view. Interfaces, 4(3), 28-36. https://doi.org/10.1287/inte.4.3.28

Geunes, J., \& Su, Y. (2020). Single-period assortment and stock-level decisions for dual sales channels with capacity limits and uncertain demand. International Journal of Production Research, 58(18), 55795600. https://doi.org/10.1080/00207543.2019.1693648

Granovetter, M. S. (1973). The strength of weak ties. American Journal of Sociology, 78(6), 1360-1380. https://doi.org/10.1086/225469

Grover, P., Kar, A. K., \& Dwivedi, Y. K. (2020). Understanding artificial intelligence adoption in operations management: Insights from the review of academic literature and social media discussions. Annals of Operations Research. https://doi.org/10.1007/s10479-020-03683-9

Guo, X., Kapucu, N., \& Huang, J. (2021). Examining resilience of disaster response system in response to COVID-19. International Journal of Disaster Risk Reduction, 59, 102239. https://doi.org/10.1016/j. ijdrr.2021.102239

Gupta, M., Shoja, A., \& Mikalef, P. (2021). Toward the understanding of national culture in the success of non-pharmaceutical technological interventions in mitigating COVID-19 pandemic. Annals of Operations Research. https://doi.org/10.1007/s10479-021-03962-Z

Gupta, S., Altay, N., \& Luo, Z. (2019). Big data in humanitarian supply chain management: A review and further research directions. Annals of Operations Research, 283(1-2), 1153-1173. https://doi.org/10. 1007/s10479-017-2671-4

Hao-Chih, L., Kai-Yue, D., Lin, B., Liu, C., Robson, P., Chung, M., Bernheim, A., Calcagno, C., Li, S., Hong, S., Long, Q., Steinberger, S., Jacobi, A., Liu, F., Little, B., Fayad, Z., \& Yang, Y. (2020). Artificial intelligence-enabled rapid diagnosis of patients with COVID-19. Nature Medicine, 26(8), 1224-1228. 
Hassan, N. M., \& Abbasi, M. N. (2021). A review of supply chain integration extents, contingencies and performance: A post Covid-19 review. Operations Research Perspectives. https://doi.org/10.1016/j. orp.2021.100183

Hensmans, M. (2021). Exploring the dark and bright sides of Internet democracy: Ethos-reversing and ethos-renewing digital transformation. Technological Forecasting and Social Change, 168, 120777. https://doi.org/10.1016/j.techfore.2021.120777

John, H. (2006). Studying complex adaptive systems. Journal of Systems Science and Complexity, 19(1), $1-8$.

Horne, J. F. (1997). The coming age of organizational resilience. Business Forum, 22, 24-29.

Hosseini, S., \& Ivanov, D. (2020). Bayesian networks for supply chain risk, resilience and ripple effect analysis: A literature review. Expert Systems with Applications, 161, 113649. https://doi.org/10.1016/j. eswa.2020.113649

Hwang, H., \& Höllerer, M. A. (2020). The COVID-19 crisis and its consequences: Ruptures and transformations in the global institutional fabric. Journal of Applied Behavioral Science, 56(3), 294-300. https://doi.org/10.1177/0021886320936841

Ivanov, D. (2020a). Predicting the impacts of epidemic outbreaks on global supply chains: A simulationbased analysis on the coronavirus outbreak. Transportation Research Part E, 136(March), 101922. https://doi.org/10.1016/j.tre.2020.101922

Ivanov, D. (2020b). Viable supply chain model: Integrating agility, resilience and sustainability perspectives-lessons from and thinking beyond the COVID-19 pandemic. Annals of Operations Research. https://doi.org/10.1007/s10479-020-03640-6

Ivanov, D. (2021). Exiting the COVID-19 pandemic: after-shock risks and avoidance of disruption tails in supply chains. Annals of Operations Research. https://doi.org/10.1007/s10479-021-04047-7

Ivanov, D., \& Dolgui, A. (2020). A digital supply chain twin for managing the disruption risks and resilience in the era of Industry 4. 0. Production Planning and Control. https://doi.org/10.1080/09537287. 2020.1768450

Jiang, X., Coffee, M., Bari, A., Wang, J., Jiang, X., Huang, J., Shi, J., Dai, J., Cai, J., Zhang, T., Wu, Z., He, G., \& Huang, Y. (2020). Towards an Artificial Intelligence Framework for Data-Driven Prediction of Coronavirus Clinical Severity. Computers Materials \& Continua., 62(3), 537-551. https://doi.org/10. 32604/cmc.2020.010691

Kafeza, E., Makris, C., Rompolas, G., \& Al-Obeidat, F. (2020). Behavioral and migration analysis of the dynamic customer relationships on Twitter. Information Systems Frontiers. https://doi.org/10.1007/ s10796-020-10033-4

Kapoor, K. K., Tamilmani, K., Rana, N. P., Patil, P., Dwivedi, Y. K., \& Nerur, S. (2018). Advances in social media research: Past, present and future. Information Systems Frontiers, 20(3), 531-558. https://doi. org/10.1007/s10796-017-9810-y

Karpen, I. O., \& Conduit, J. (2020). Engaging in times of COVID-19 and beyond: Theorizing customer engagement through different paradigmatic lenses. Journal of Service Management, 31(6), 11631174. https://doi.org/10.1108/JOSM-05-2020-0156

Katsaliaki, K., Galetsi, P., \& Kumar, S. (2021). Supply chain disruptions and resilience: A major review and future research agenda. Annals of Operations Research. https://doi.org/10.1007/s10479-020-03912-1

Kumar, A., Mangla, S. K., Kumar, P., \& Song, M. (2021a). Mitigate risks in perishable food supply chains: Learning from COVID-19. Technological Forecasting and Social Change. https://doi.org/10.1016/j. techfore.2021.120643

Kumar, P., \& Singh, R. K. (2021). Application of Industry technologies for effective coordination in humanitarian supply chains: A strategic approach. Annals of Operations Research. https://doi.org/10.1007/ s10479-020-03898-w

Kumar, S., Xu, C., Ghildayal, N., Chandra, C., \& Yang, M. (2021b). Social media effectiveness as a humanitarian response to mitigate influenza epidemic and COVID-19 pandemic. Annals of Operations Research. https://doi.org/10.1007/s10479-021-03955-y

Lawrence, P. R., \& Lorsch, J. W. (1967). Differentiation and integration in complex organizations. Administrative Science Quarterly, 12(1), 1. https://doi.org/10.2307/2391211

Lee, S. M., \& Trimi, S. (2021). Convergence innovation in the digital age and in the COVID-19 pandemic crisis. Journal of Business Research, 123, 14-22. https://doi.org/10.1016/j.jbusres.2020.09.041

Li, D., Wang, D., Dong, J., Wang, N., Huang, H., Xu, H., \& Xia, C. (2020a). False-negative results of realtime reverse-transcriptase polymerase chain reaction for severe acute respiratory syndrome coronavirus 2: Role of deep-learning-based CT diagnosis and insights from two cases. Korean Journal of Radiology, 21(4), 505. https://doi.org/10.3348/kjr.2020.0146 
Li, S., Wang, Y., Xue, J., Zhao, N., \& Zhu, T. (2020b). The impact of covid-19 epidemic declaration on psychological consequences: A study on active weibo users. International Journal of Environmental Research and Public Health. https://doi.org/10.3390/ijerph17062032

Li, Y., Chen, K., Collignon, S., \& Ivanov, D. (2020c). Ripple effect in the supply chain network: Forward and backward disruption propagation, network health and firm vulnerability. European Journal of Operational Research. https://doi.org/10.1016/j.ejor.2020.09.053

Manero, A., Smith, P., Koontz, A., Dombrowski, M., Sparkman, J., Courbin, D., \& Chi, A. (2020). Leveraging 3D printing capacity in times of crisis: Recommendations for COVID-19 distributed manufacturing for medical equipment rapid response. International Journal of Environmental Research and Public Health, 17(13), 1-17. https://doi.org/10.3390/ijerph17134634

Mao, C., Koide, R., Brem, A., \& Akenji, L. (2020). Technology foresight for social good: Social implications of technological innovation by 2050 from a global expert survey. Technological Forecasting and Social Change, 153, 119914. https://doi.org/10.1016/j.techfore.2020.119914

Marin-Garcia, J. A., Garcia-Sabater, J. P., Ruiz, A., Maheut, J., \& Garcia-Sabater, J. (2020). Operations management at the service of health care management: Example of a proposal for action research to plan and schedule health resources in scenarios derived from the COVID-19 outbreak. Journal of Industrial Engineering and Management, 13(2), 213-227. https://doi.org/10.3926/jiem.3190

McCall, B. (2020). COVID-19 and artificial intelligence: protecting health-care workers and curbing the spread. The Lancet Digital Health, 2(4), e166-e167. https://doi.org/10.1016/s2589-7500(20) 30054-6

McKinsey. (2020). The COVID-19 recovery will be digital: A plan for the first 90 days. https://www. mckinsey.com/business-functions/mckinsey-digital/our-insights/the-covid-19-recovery-will-bedigital-a-plan-for-the-first-90-days

Mileder, L. P., Schüttengruber, G., Prattes, J., \& Wegscheider, T. (2020). Simulation-based training and assessment of mobile pre-hospital SARS-CoV-2 diagnostic teams in Styria. Austria Medicine, 99(29), e21081. https://doi.org/10.1097/MD.0000000000021081

Mishra, D., Gunasekaran, A., Papadopoulos, T., \& Childe, S. J. (2018). Big data and supply chain management: A review and bibliometric analysis. Annals of Operations Research, 270(1-2), 313-336. https://doi.org/10.1007/s10479-016-2236-y

Mitręga, M., \& Choi, T. M. (2021). How small-and-medium transportation companies handle asymmetric customer relationships under COVID-19 pandemic: A multi-method study. Transportation Research Part E: Logistics and Transportation Review. https://doi.org/10.1016/j.tre.2021.102249

Nandi, S., Sarkis, J., Hervani, A., \& Helms, M. (2020). Do blockchain and circular economy practices improve post COVID-19 supply chains? A resource-based and resource dependence perspective. Industrial Management and Data Systems, 121(2), 333-363. https://doi.org/10.1108/ IMDS-09-2020-0560

Nunes, R. M. S., \& Pereira, S. C. F. (2021). Intellectual structure and trends in the humanitarian operations field. Annals of Operations Research. https://doi.org/10.1007/s10479-021-04022-2

Ozturk, T., Talo, M., Yildirim, E. A., Baloglu, U. B., Yildirim, O., \& Rajendra Acharya, U. (2020). Automated detection of COVID-19 cases using deep neural networks with X-ray images. Computers in Biology and Medicine. https://doi.org/10.1016/j.compbiomed.2020.103792

Pacheco, J., \& Laguna, M. (2020). Vehicle routing for the urgent delivery of face shields during the COVID-19 pandemic. Journal of Heuristics. https://doi.org/10.1007/s10732-020-09456-8

Pan, S. L., \& Zhang, S. (2020). From fighting COVID-19 pandemic to tackling sustainable development goals: An opportunity for responsible information systems research. International Journal of Information Management. https://doi.org/10.1016/j.ijinfomgt.2020.102196

Papagiannidis, S., Harris, J., \& Morton, D. (2020). WHO led the digital transformation of your company? A reflection of IT related challenges during the pandemic. International Journal of Information Management. https://doi.org/10.1016/j.ijinfomgt.2020.102166

Queiroz, M. M., Ivanov, D., Dolgui, A., \& Fosso Wamba, S. (2020a). Impacts of epidemic outbreaks on supply chains: Mapping a research agenda amid the COVID-19 pandemic through a structured literature review. Annals of Operations Research. https://doi.org/10.1007/s10479-020-03685-7

Queiroz, M. M., Fosso Wamba, S., Machado, M. C., \& Telles, R. (2020b). Smart production systems drivers for business process management improvement: An integrative framework. Business Process Management Journal, 26(5), 1075-1092. https://doi.org/10.1108/BPMJ-03-2019-0134

Ranjbari, M., Shams Esfandabadi, Z., Zanetti, M. C., Scagnelli, S. D., Siebers, P. O., Aghbashlo, M., Peng, W., Quatraro, F., \& Tabatabaei, M. (2021). Three pillars of sustainability in the wake of COVID-19: A systematic review and future research agenda for sustainable development. Journal of Cleaner Production. https://doi.org/10.1016/j.jclepro.2021.126660 
Rotolo, D., Hicks, D., \& Martin, B. R. (2015). What is an emerging technology? Research Policy, 44(10), 1827-1843. https://doi.org/10.1016/j.respol.2015.06.006

Ruel, S., El Baz, J., Ivanov, D., \& Das, A. (2021). Supply chain viability: Conceptualization, measurement, and nomological validation. Annals of Operations Research. https://doi.org/10.1007/ s10479-021-03974-9

Sahu, C. K., Young, C., \& Rai, R. (2020). Artificial intelligence (AI) in augmented reality (AR)-assisted manufacturing applications: A review. International Journal of Production Research. https://doi.org/ 10.1080/00207543.2020.1859636

Santosh, N., Joseph, S., Aref Aghaei, H., \& Marilyn, M. H. (2021). Redesigning supply chains using blockchain-enabled circular economy and COVID-19 experiences. Sustainable Production and Consumption, 27, 10-21.

Sarkis, J. (2020). Supply chain sustainability: Learning from the COVID-19 pandemic. International Journal of Operations and Production Management, 41(1), 63-73. https://doi.org/10.1108/ IJOPM-08-2020-0568

Sarkis, J., Cohen, M. J., Dewick, P., \& Schröder, P. (2020). A brave new world: Lessons from the COVID19 pandemic for transitioning to sustainable supply and production. Resources, Conservation and Recycling. https://doi.org/10.1016/j.resconrec.2020.104894

Singh, S., Kumar, R., Panchal, R., \& Tiwari, M. K. (2020). Impact of COVID-19 on logistics systems and disruptions in food supply chain. International Journal of Production Research. https://doi.org/10. 1080/00207543.2020.1792000

Sinha, A., Kumar, P., Rana, N. P., Islam, R., \& Dwivedi, Y. K. (2019). Impact of internet of things (IoT) in disaster management: A task-technology fit perspective. Annals of Operations Research, 283(1-2), 759-794. https://doi.org/10.1007/s10479-017-2658-1

Sinha, P., Kumar, S., \& Chandra, C. (2021). Strategies for ensuring required service level for COVID-19 herd immunity in indian vaccine supply chain. European Journal of Operational Research. https:// doi.org/10.1016/j.ejor.2021.03.030

Siriwardhana, Y., De Alwis, C., Gur, G., Ylianttila, M., \& Liyanage, M. (2020). The fight against COVID19 pandemic with 5G technologies. IEEE Engineering Management Review. https://doi.org/10.1109/ EMR.2020.3017451

Sodhi, M., Tang, C., \& Willenson, E. (2021). Research Opportunities in Preparing Supply Chains of Essential Goods for Future Pandemics. International Journal of Production Research. https://doi.org/10. 1080/00207543.2021.1884310

Spanaki, K., Sivarajah, U., Fakhimi, M., Despoudi, S., \& Irani, Z. (2021). Disruptive technologies in agricultural operations: A systematic review of AI-driven AgriTech research. Annals of Operations Research. https://doi.org/10.1007/s10479-020-03922-z

Surabhi, V., \& Anders, G. (2020). Investigating the emerging COVID-19 research trends in the field of business and management: A bibliometric analysis approach. Journal of Business Research, 118, 253-261.

Teece, D. J., Pisano, G., \& Shuen, A. M. Y. (1997). Dynamic capabilities and strategic management. Strategic Management Journal, 18(7), 509-533.

Teece, D., \& Pisano, G. (1994). The dynamic capabilities of firms: An introduction. Industrial and Corporate Change, 3(3), 537-556. https://doi.org/10.1093/icc/3.3.537-a

Van de Ven, A. H., Ganco, M., Hinings, C. R., \& (BOB). . (2013). Returning to the frontier of contingency theory of organizational and institutional designs. Academy of Management Annals, 7(1), 393-440. https://doi.org/10.5465/19416520.2013.774981

van Remko, H. (2020). Research opportunities for a more resilient post-COVID-19 supply chain - closing the gap between research findings and industry practice. International Journal of Operations and Production Management, 40(4), 341-355. https://doi.org/10.1108/IJOPM-03-2020-0165

Wamba, S. F., \& Queiroz, M. M. (2020). Industry and the supply chain digitalisation: A blockchain diffusion perspective. Production Planning and Control. https://doi.org/10.1080/09537287.2020.1810756

Wernerfelt, B. (1984). A resource-based view of the firm. Strategic Management Journal, 5(2), 171-180. https://doi.org/10.1002/smj.4250050207

Wilson, S. (2020). The pandemic, the acceleration of digital transformation and the impact on cyber security. Computer Fraud and Security, 2020(12), 13-15. https://doi.org/10.1016/S1361-3723(20)30128-7

Wynants, L., Van Calster, B., Collins, G. S., Riley, R. D., Heinze, G., Schuit, E., Bonten, M. M. J., Dahly, D. L., Damen, J. A., Debray, T. P. A., de Jong, V. M. T., De Vos, M., Dhiman, P., Haller, M. C., Harhay, M. O., Henckaerts, L., Heus, P., Kammer, M., Kreuzberger, N., \& van Smeden, M. (2020). Prediction models for diagnosis and prognosis of covid-19: Systematic review and critical appraisal. BMJ. https://doi.org/10.1136/bmj.m1328 
Yan, L., Zhang, H.-T., Goncalves, J., Xiao, Y., Wang, M., Guo, Y., Sun, C., Tang, X., Jing, L., Zhang, M., Huang, X., Xiao, Y., Cao, H., Chen, Y., Ren, T., Wang, F., Xiao, Y., Huang, S., Tan, X., \& Yuan, Y. (2020). An interpretable mortality prediction model for COVID-19 patients. Nature Machine Intelligence, 2(5), 283-288. https://doi.org/10.1038/s42256-020-0180-7

Yu, W., Zhao, G., Liu, Q., \& Song, Y. (2021). Role of big data analytics capability in developing integrated hospital supply chains and operational flexibility: An organizational information processing theory perspective. Technological Forecasting and Social Change. https://doi.org/10.1016/j.techfore.2020. 120417

Publisher's Note Springer Nature remains neutral with regard to jurisdictional claims in published maps and institutional affiliations. 